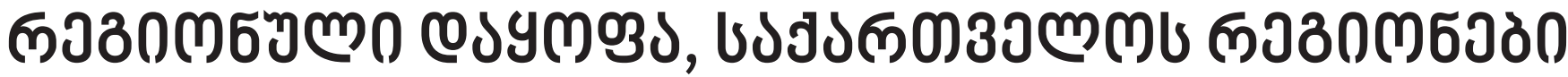

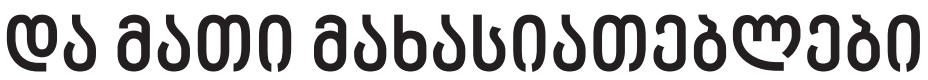

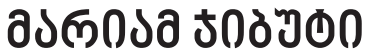

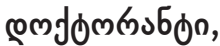

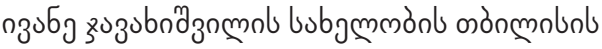

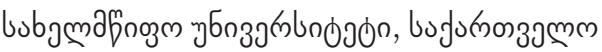 \\ mariami.jibuti382@eab.tsu.edu.ge
}

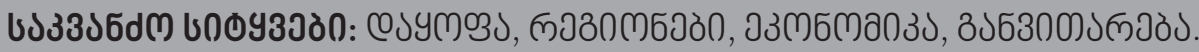

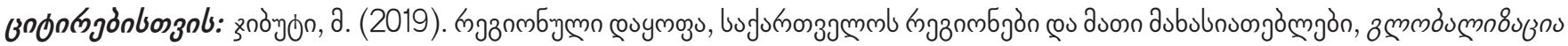
cou ono86jbn, №8, 33. 126-129. https://doi.org/10.35945/gb.2019.08.016

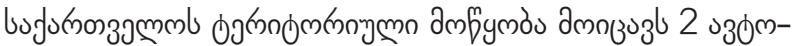

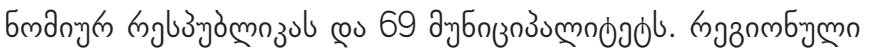

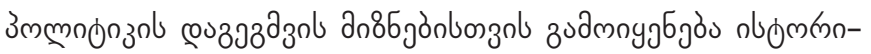

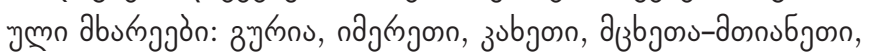

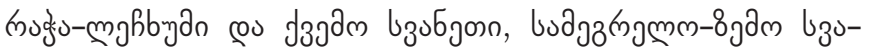

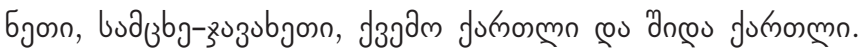

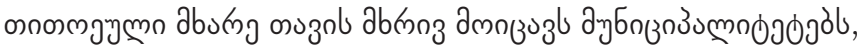

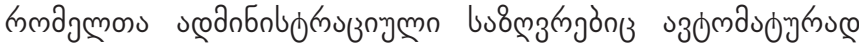
зublu8

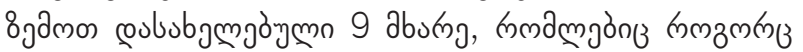

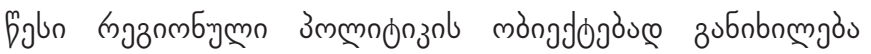

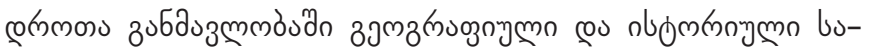

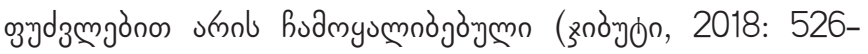

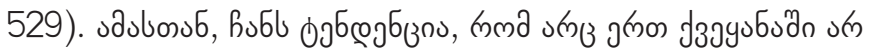

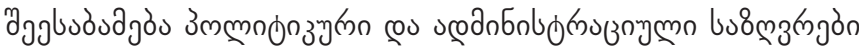

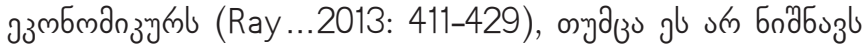

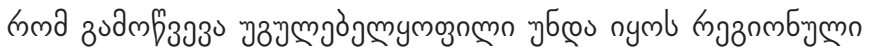

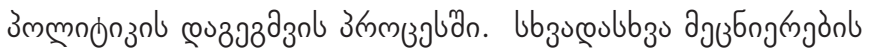

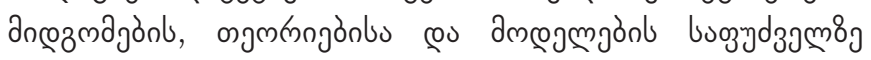

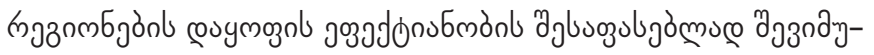

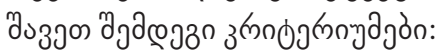

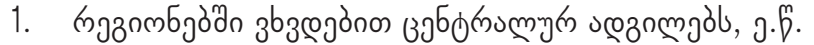

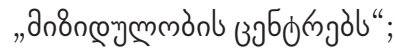

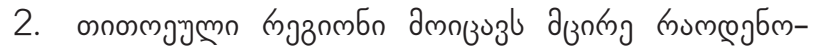

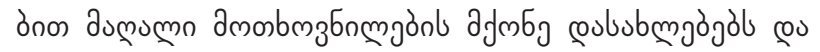

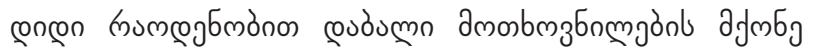
coububmajajl;

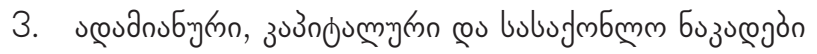

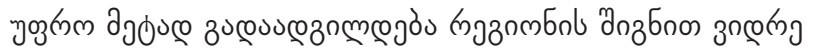

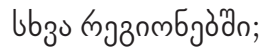

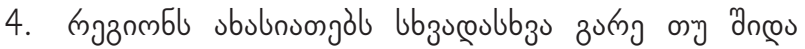

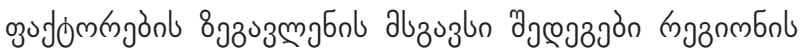

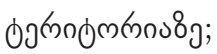

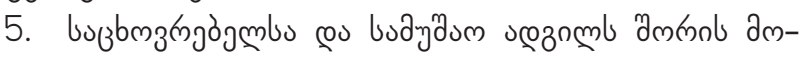

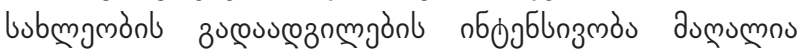

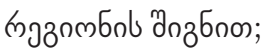

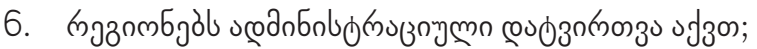

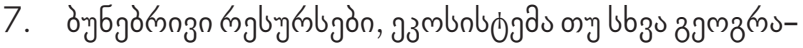

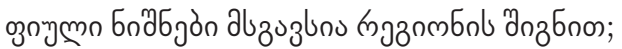

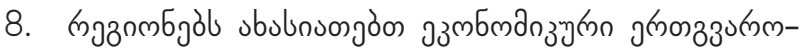
3ढjò;

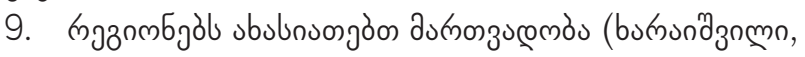
2003: 88).

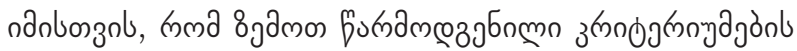

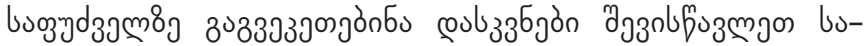

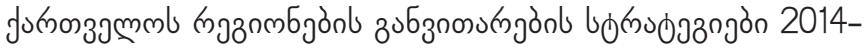

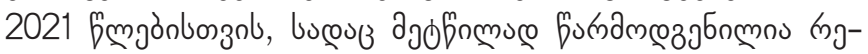

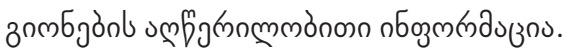

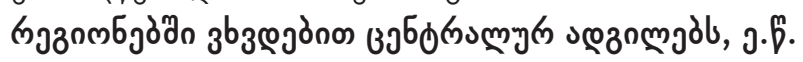

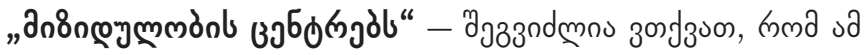

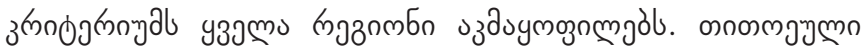

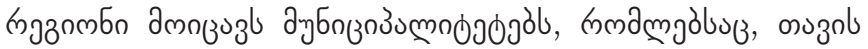

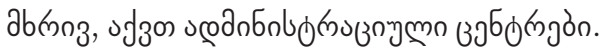

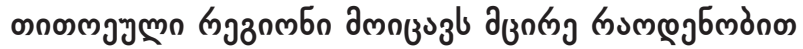
aumumn дmorbm

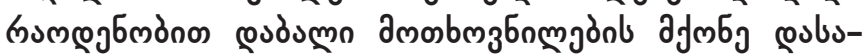

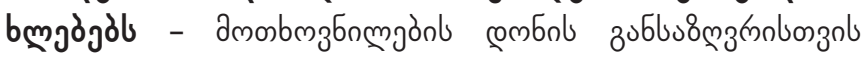

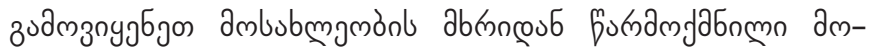

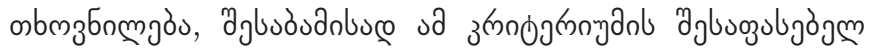

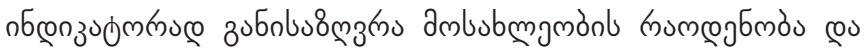




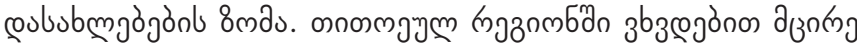

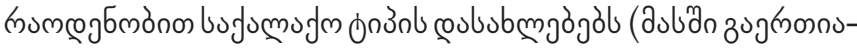

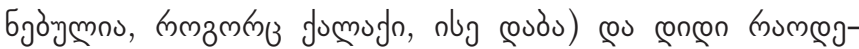

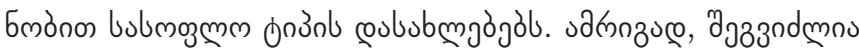

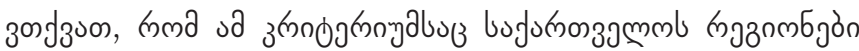

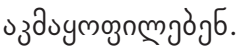

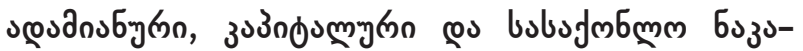

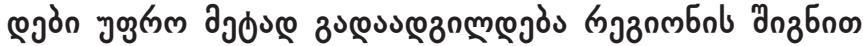

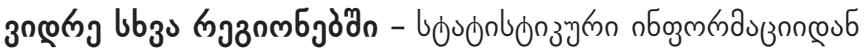

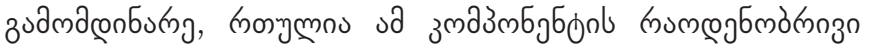

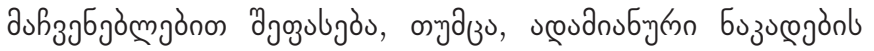

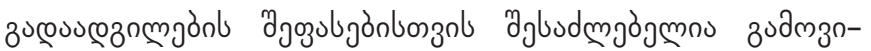

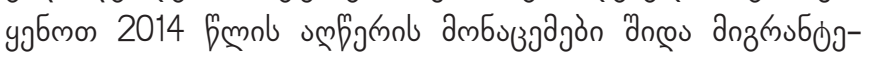

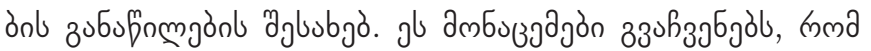

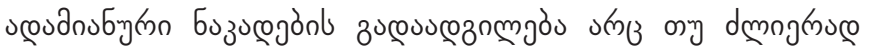

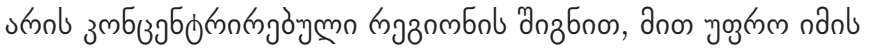

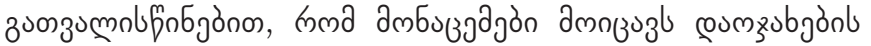

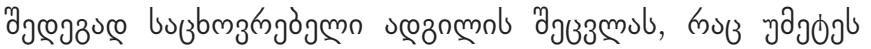

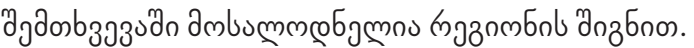

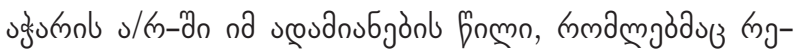

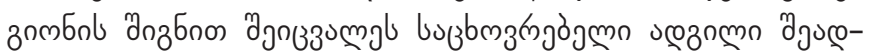

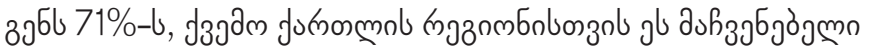

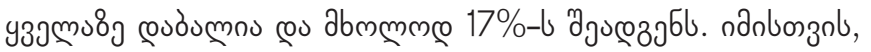

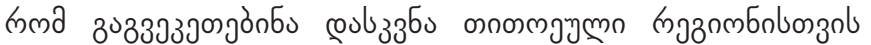

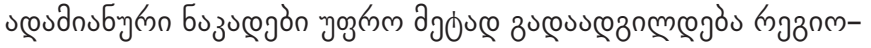
бn b

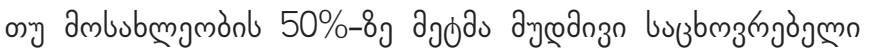

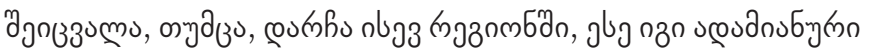

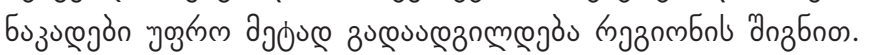

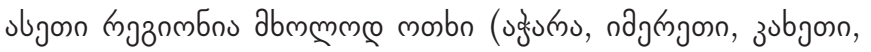

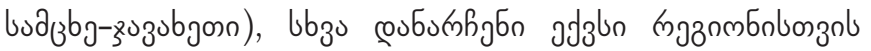

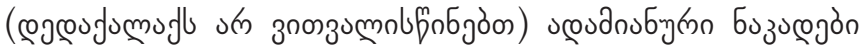

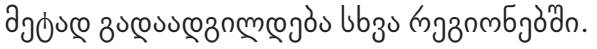

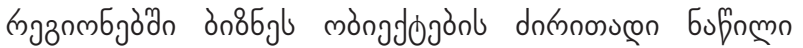

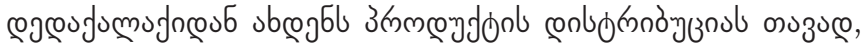

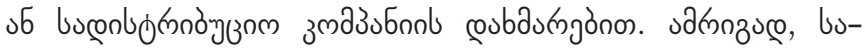

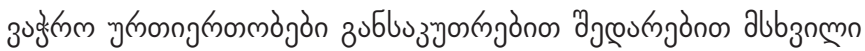

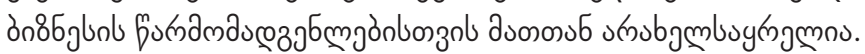

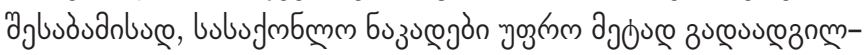

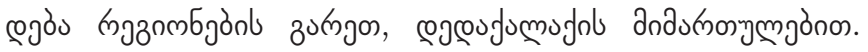

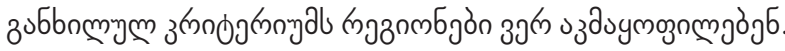

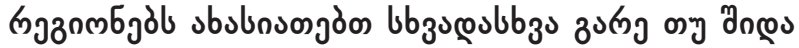

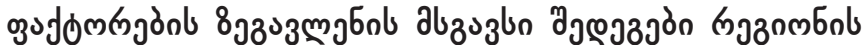

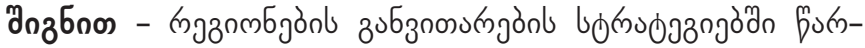

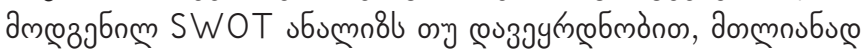

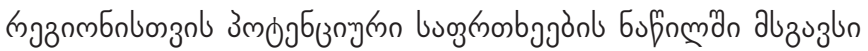

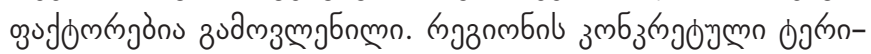

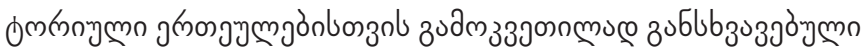

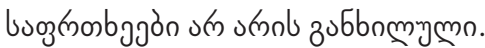

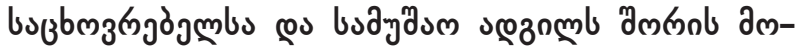

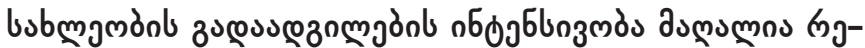

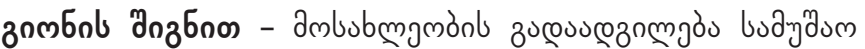

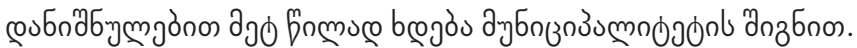

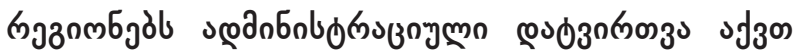

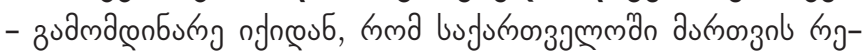

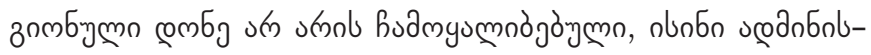

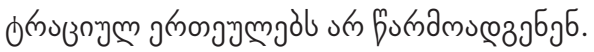

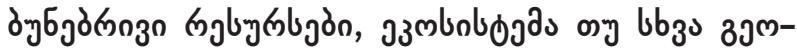

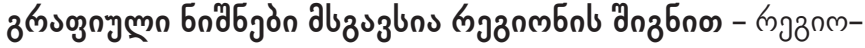

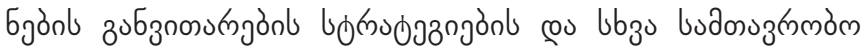

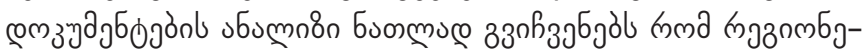

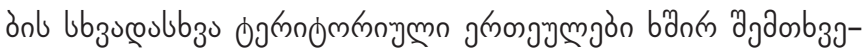

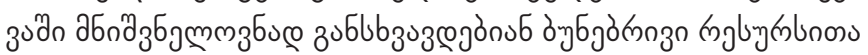

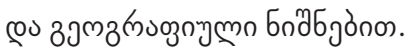

๓лзвмб

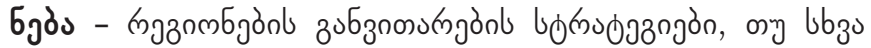

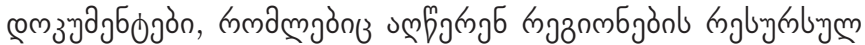

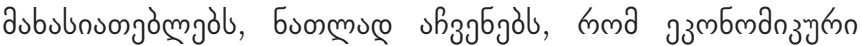

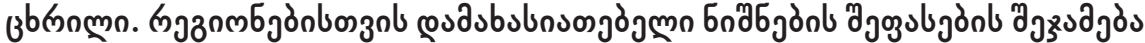

\begin{tabular}{|c|c|}
\hline 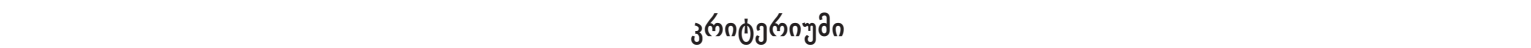 & əวœэว \\
\hline 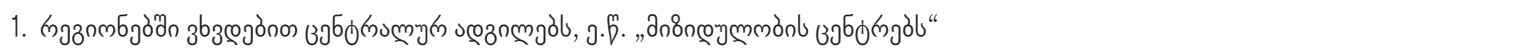 & V \\
\hline 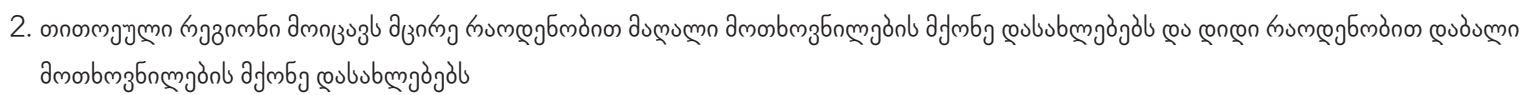 & $\vee$ \\
\hline 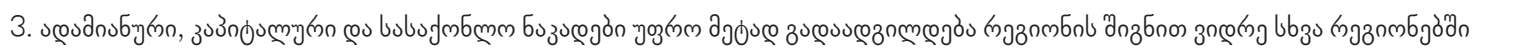 & - \\
\hline 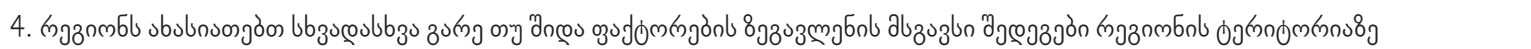 & $\vee$ \\
\hline 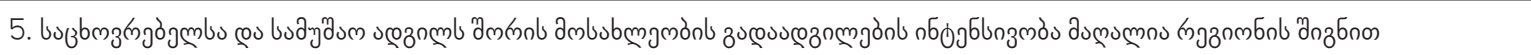 & V \\
\hline 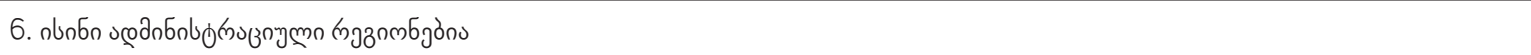 & - \\
\hline 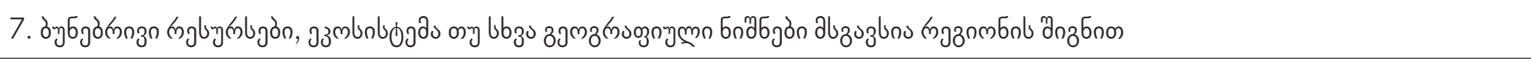 & - \\
\hline 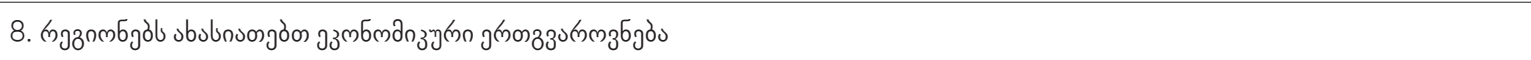 & - \\
\hline 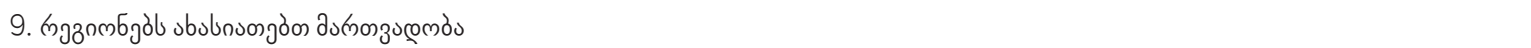 & - \\
\hline
\end{tabular}




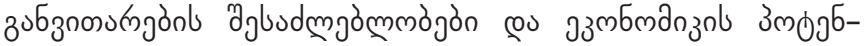

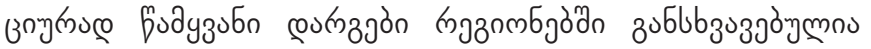

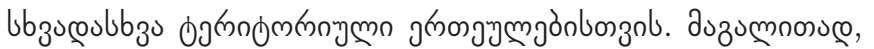
zy

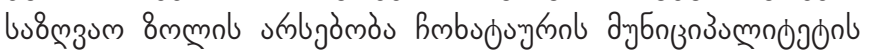

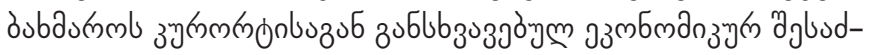

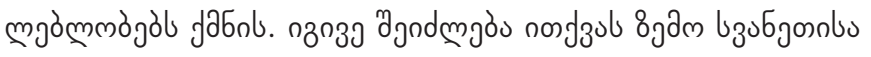

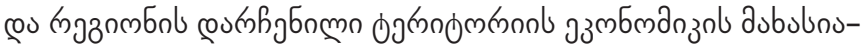

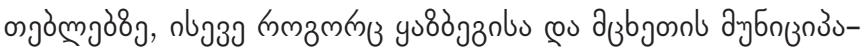
modjojoby.

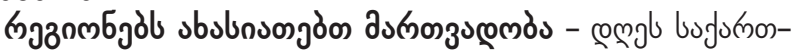

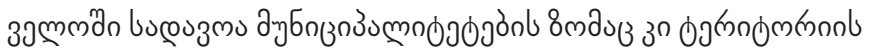

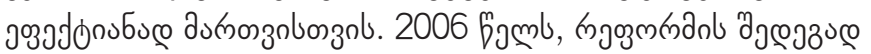

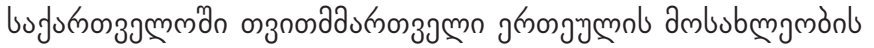

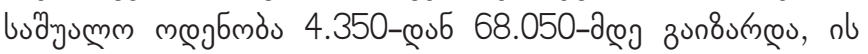

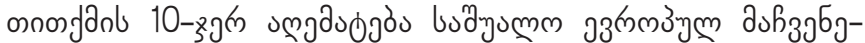

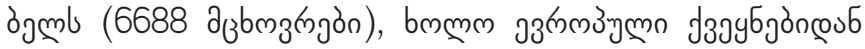

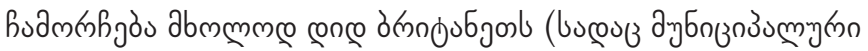

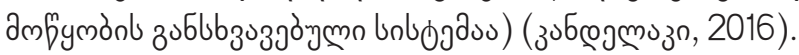

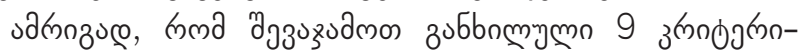

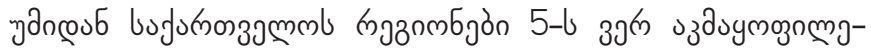

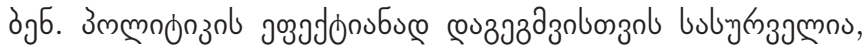

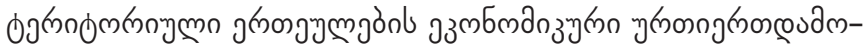
зпœ9

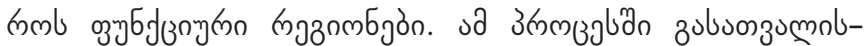

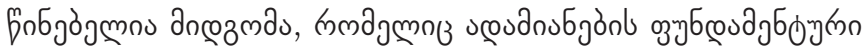

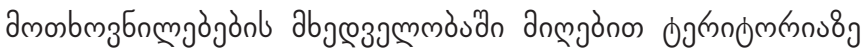

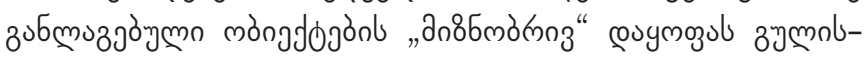

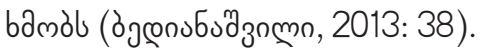

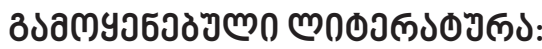

1. Regional Development Programme of Georgia 2018-2021, Government of Georgia (In Georgian).http://www.mrdi.gov. ge/sites/default/files/2018-2021_clebis_sakartvelos_regionuli_ganvitarebis_programa_0.pdf

2. Bedianashvili, G. (2013). The Territorial Structure of the Country and the System Framework of Local Government Reform [Kveknis Teritoriuli Striktura da Adgilobrivi Tvitmmartvelobis Repormis Sistemuri Sapudzvlebi]. Journal "Ekonomisti", October-November \#5, Tbilisi. (In Georgian).

3. Bedianashvili, G. (1990). Complex Socio-Economic Development of Region: Target and Resource Aspects [Regionis kompleksuri socialur-ekonomikuri ganvitareba: miznobrivi da resursuli aspeqtebi]. Metsnierteba, Tbilisi. (In Georgian).

4. Kandelaki, K. Abuladze, M. (2016). Administrative-Territorial Reform: Needs and View of the Future [AdministraciulTeritoriuli Reporma: Sachiroeba da Momavlis Khedva]. International Center for Civic Culture \& Green Caucasus, Tbilisi. (In Georgian). http://www.ivote.ge/GREENCAUCASUS/wp-content/uploads/2016/09/Concept-of-Territorial-Reform.pdf

5. Kharaishvili, E. (2003). Regional Economics [Regionuli ekonomika]. Tbilisi, 88. (In .Georgian).

6. Jibuti, M. (2008). Challenges of Regional Economic Development in Georgia [Regionebis Ekonomikuri Ganvitarebis Gamotsvevebi Sakartveloshi]. Challenges of Globalization in Economics and Business, III International Scientific Conference, Proceedings, Tbilisi, October, 5. (In Georgian).

7. Cremer, H. Pestieau, P. (2004). Factor Mobility and Redistribution, Handbook of Regional and Urban Economics, Volume \#4, Cities and Geography, Netherlands.

8. Christaller, W. (1966). Central Places in Southern Germany, Translated by Carlisle W. Baskin.

9. Combes, P. Overman, H.G. (2004). The Spatial Distribution of Economic Activities in the European Union, Handbook of Regional and Urban Economics, Volume \#4, Cities and Geography, Netherlands.

10. Dawkins, C.J. (2003). Regional Development Theory: Conceptual Foundations, Classic Works, and Recent Developments, 131-172. Journal of Planning Literature.

11. Epple, D. Nechyba, T. (2004). Fiscal Decentralization, Handbook of Regional and Urban Economics, Volume \#4, Cities and Geography, Netherlands.

12. Fox, A.K. Krishna, T.K. (1994). The functional economic area: Delineation and Implications for Economic Analysis and Police.

13. Fujita, M. Thisse, J. (2013). Economics of Agglomeration: Cities, Industrial Location, and Globalization, Second Edition.

14. Fujita, M. Mori, T. Henderson, J.V. Kameroto, Y. (2004). Spatial Distribution of Economic Activities in Japan and China, Handbook of Regional and Urban Economics, Volume \#4, Cities and Geography, Netherlands.

15. Fujita, M. Krugman, P. Venables, A. J. The Spatial Economy: Cities, Regions, and International Trade, The MIT Press Cambridge, Massachusetts London, England, 2000.

16. Ray, D.M. Lamarche, R.H. MacLachlan, I.R. (2013). Restoring the "Regional" to Regional Policy: A Regional Typology of Western Canada, Canadian Public Policy, University of Toronto Press, Volume 39, No. 3, September, pp. $411-429$.

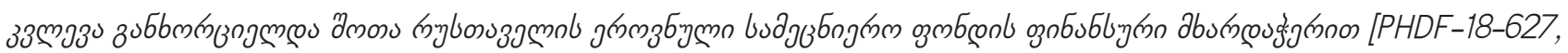

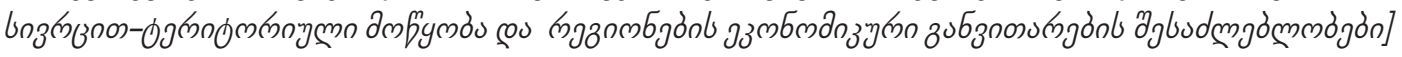




\title{
ADMINISTRATIVE DIVISION, REGIONS OF GEORGIA AND THEIR CHARACTERISTICS
}

\author{
MARIAM JIBUTI \\ PhD Student, \\ Ivane Javakhishvili Tbilisi State University, Georgia \\ mariami.jibuti382@eab.tsu.edu.ge
}

\section{KEYWORDS: DIVISION, REGIONS, ECONOMIC, DEVELOPMENT.}

For citation: Jibuti, M. (2019). Administrative Division, Regions of Georgia and their Characteristics, Globalization And Business, №8, pp. 126-129. https://doi.org/10.35945/gb.2019.08.016

\section{SUMMARY}

Georgia has a one-level system of governance. Regional level of governance is not defined by law. However, historical areas/regions are objects for policy planning (Jibuti, 2018: 526-529). Effectiveness of country's regional division had not been assessed or studied in terms of economic development planning purpose. Our research aimed to define criteria for such assessment and make an evaluation. These criteria were formed based on study of different scientific approaches, theories and models. Criteria which were used to assess effectiveness of regional division in Georgia are following: (1) Regions have central places, "core/leading centers"; (2) Each region has settlements with high demand and settlements with relatively low demand; (3) Human, capital and product flows are more intensive within the region rather than with other regions; (4) Influence of internal or external factors are similar within the territory of a region; (5) Intensity of commuting is high within a region; (6) Regions are administrative units; (7) Natural resources, ecosystem or other geographical characteristics are similar within a region; (8) Economic conditions are somewhat similar within a region; (9) It is possible to manage or govern a region (Kharaishvili, 2003: 88).

In order to make an assessment based on the abovementioned criteria we studied development strategies of all regions (that are under control of Georgian government, excluding the capital) in Georgia for 2014-2021, and analyzed regional statistics. Study results show that 5 criteria out of 9 are not true for Georgian regions. Effectiveness of the regional policy will increase if the Georgian government establishes functional regions, taking into account basic needs of people inhabited in the specific areas (Beadianashvili, 2013: 38).

This work was supported by Shota Rustaveli National Science Foundation (SRNSF) [PHDF-18-627, Spatial-Territorial Planning and Economic Development Opportunities of Regions] 